\title{
Influence of the Relative Position of Impeller and Baffles in Multiple Reference Frame Simulation of Gas-Liquid Mixing in Stirred Tank
}

\author{
D. V. Gradov, A. Laari, and T. Koiranen
}

\begin{abstract}
In this paper analysis is carried out on the response of hydrodynamics to the relative position of stirrer and baffles when Multiple Reference Frame (MRF) technique is used to simulate a mixing tank of single and multiphase systems. A typical stirred reactor equipped with four baffles and a six-blade Rushton turbine is studied. Operational conditions of constant speed $(5 \mathrm{rps})$ and $2.653 \cdot 10^{-4} \mathrm{~m} / \mathrm{s}$ gas superficial velocity with constant bubble size $(1 \mathrm{~mm})$ were applied. The simulations were performed in ANSYS Workbench (FLUENT) 16.0 with four different angles of impeller blade with regard to baffles. The results were averaged over two utmost and all four impeller blade positions and validated against experimental data, produced by means of Particle Image Velocimetry. Comparison of the simulated and experimental results was executed using profiles of velocity components along sampled lines, representing various zones in the vessel. The impeller angle of rotation with respect to the position of baffles was found to contribute to the overall reactor hydrodynamics especially in multiphase systems. Averaging of the simulation results over all impeller positions was shown to enhance the accuracy of the simulated results of single phase and multiphase mixing hydrodynamics.
\end{abstract}

Index Terms-Stirred tank, multiphase, CFD, PIV, validation.

\section{INTRODUCTION}

Stirred tank is one of the key unit operations widely utilized in chemical industry. Its performance has direct impact on production quality, yield, and therefore overall effectiveness. That is why it is extremely important to match a certain chemical reaction to a suitable reactor and to operate it under optimal conditions. It is well known that chemical process features and performance can be forecasted and its output estimated via mathematic modeling.

Chemical reaction modeling often employs the assumption of the media to be perfectly mixed. In order to assess the hydrodynamics within a reactor it is possible to take into account different geometries and scales of operation using Computational Fluid Dynamics (CFD). The usage of Reynolds-averaged Navier-Stokes (RANS) equations is a compromise between the needs of engineering, computational time and resources available.

Since 80's stirred tank modeling has been studied in literature by many research groups such as Issa and Gosman,

Manuscript received October 15, 2015; revised March 26, 2016

D. V. Gradov is with Lappeenvanta University of Technology, Finland (e-mail: dmitry.gradov@lut.fi).

A. Laari and T. Koiranen are with Lappeenvanta University of Technology, Finland (e-mail: arto.laari@lut.fi, tuomas.koiranen@lut.fi).
Harvey and Greaves, Lane et al., Vivek et al., Laakkonen et al., Bai et al., and Huang et al. among many others [1]-[7]. Liquid mixing is described numerically mainly via two-equation models of RANS family due to affordable computational load. For this reason, the Large Eddy Simulation approach has fewer applications in multiphase mixing regardless the fact that it shows higher accuracy compared to experimental data. Aubin et al. [8] and Vlachakis [9] claim that the impeller interaction with baffles has a periodic character that allows to assume the stirred reactor to be in a steady state. Two main approaches to treat the impeller geometry are generally used when stirred tank is modeled. These are the Sliding Mesh (SM) and the Multiple Reference Frame approaches. The MRF simulation aids in saving time without losses in accuracy compared to transient SM approach where the actual geometry of agitator is moving. According to Marshal and Bakker in "Computational Fluid Mixing" [10] mixing tank simulations with the "frozen rotor" (MRF) method using the outermost impeller positions and averaging the results should be carried out to produce accurate results. This suggestion has not been usually followed in the published literature. Therefore, the influence of the impeller blade position relative to baffles on the overall hydrodynamics in a vessel equipped with four baffles is studied in this work and evaluated qualitatively and numerically by averaging the simulation data.

There are several experimental methods for validating numerical simulation data of hydrodynamics. They could be grouped as intrusive methods, which are mainly based on Constant Temperature Anemometry, for example Hot Wire Anemometry (HWA) [11], and non-intrusive ones, such as Laser Doppler Velocimetry (LDA) [12] and Particle Image Velocimetry (PIV) [13]. The PIV technique provides velocity vector fields in two-dimensional space that is used in this study.

The present paper is dedicated to baffle-impeller interaction assessment and results averaging procedure for stirred reactor when the Multiple Reference Frame method is applied. The single and multiphase simulation results of averaging are validated against experimental data obtained by means of PIV.

\section{MATERIALS AND METHODS}

\section{A. Object of Study}

A round bottom stirred reactor equipped with baffles and a Rushton turbine is the object of study in the current research. The dimensions of the reactor are presented in Table I. The 
vessel is made of glass and sealed in a plexiglass box for conducting PIV tests. The clearance of the six-blade impeller from the tank bottom was kept the same as its diameter. The gas inlet sparger was mounted in the very center of the vessel bottom. The operational conditions were $300 \mathrm{rpm}$ of rotational speed and $0.12 \mathrm{vvm}$ for gas flow rate. In order to examine the influence of the blades positioning far from the impeller, the liquid height was twice the tank diameter. The schematic view of the reactor together with velocity profile sampling strategy is illustrated in Fig. 1.

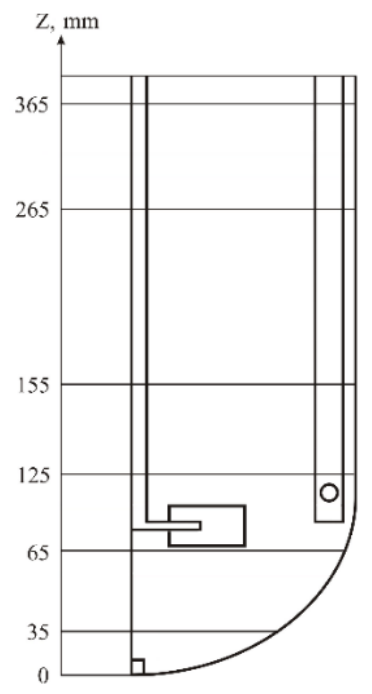

Fig. 1. Mixing reactor and schematic representation of profiles sampling positions.

\section{B. Validation Technique}

Particle Image Velocimetry is widely used for fluid flow study and its results are frequently used to validate simulation results [1], [9], [11], [14]. It produces instantaneous velocity vector field in $2 \mathrm{D}$ or $3 \mathrm{D}$ in case of stereographic PIV. By means of fast laser pulsing it is possible to track slight shift of submerged light tracers within an interrogation window of interest. A classical set-up includes cameras installed perpendicular towards a dense laser sheet produced by $\mathrm{Nd}$ :Yag laser. In the current research, two CCD cameras with a resolution of 1600 pixels $\times 1200$ pixels were used. That allows capturing $2 \mathrm{D}$ velocity fields from the whole reactor with the resolution of 188 vectors $\times 189$ vectors. The main requirements for the tracer particles are that they follow the media without disturbing the flow and reflect intensive light on certain wavelengths. It is also necessary to take into account that the studied reactor has many strongly reflecting parts which prevents the use of standard hollow glass PIV tracer particles. Therefore, $20-50 \mu \mathrm{m}$ particles made of melamine resin covered with fluorescent dye (Rhodamine B) were applied. Their usage in water flow tests has the benefit that a uniform dark background can be obtained with large camera aperture installing wave length filters on the cameras. To detect the movement of gas phase, tests were carried out with only grey filters mounted on the cameras to decrease the reflected light intensity from bubbles to protect the camera.

The position of the laser sheet was set to be at a distance of $10 \mathrm{~mm}$ in front of a baffle to maximize the area captured in a frame and to eliminate the neighboring baffle from the field of view. Each frame includes half of the reactor from the vessel wall to the agitator shaft in width and $10 \mathrm{~cm}$ in height. Therefore, six overlapping frames were taken along the vertical axes in order to capture the whole tank. The coordinates of the interrogation window were measured to extract the analogue plane from the CFD simulation results. A schematic representation of the PIV set-up is shown in Fig. 2.

\begin{tabular}{lc}
\multicolumn{2}{c}{ TABLE I: DIMENSIONS OF THE REACTOR } \\
\hline \hline Reactor dimension & Value, $\mathrm{mm}$ \\
\hline Tank diameter & 20 \\
Liquid height & 40 \\
Impeller diameter & 70 \\
Impeller blade width & 15 \\
Impeller clearance & 70 \\
Shaft diameter & 14 \\
Baffle width & 20 \\
Baffle distance from tank wall & 5 \\
Sparger height & 10 \\
Sparger width & 7 \\
\hline
\end{tabular}

The detention time between the laser pulses was adjusted individually for each position along the vertical axis so that tracer shift would not exceed the interrogation area when post-processing the data. In total, 500 frames were taken to obtain experimental results at steady state.

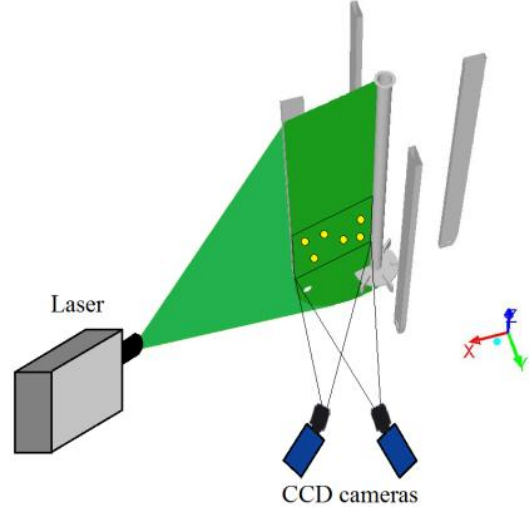

Fig. 2. Conceptual schema of two-phase PIV set-up used in the current work.

\section{Simulation Settings}

It was decided not to use geometrical symmetry and instead consider complete reactor hydrodynamics to avoid any concerns how the symmetry might affect the overall flow fields. The reactor domain was discretized by structured meshes with a maximum aspect ratio of 30 and high orthogonal quality to reduce numerical errors. Grid independence test carried out over four meshes with increasing resolution. The test showed that there is no sense to use more than 800000 elements because with further increase of mesh density velocity components values stay within $10 \%$ of the normalized root mean square error index (NRMSE) (Eq. 1) that identifies grid difference to be insignificant [15].

$$
\text { nrmse }=\frac{3}{\left(n_{\text {fine }} / n_{\text {coarse }}\right)^{p}-1} \sqrt{\frac{\sum_{i=1}^{N}\left(\phi_{1}(i)-\phi_{1}(i)\right)^{2}}{\sum_{i=1}^{n}\left(\phi_{2}\right)^{2}}} \cdot 100 \%
$$

$n_{\text {fine }}$ and $n_{\text {coarse }}$ are the number of cells in a fine and coarse grids correspondingly, $p$ represents the order of discretization, $\mathrm{N}$ is the number of samples, $\phi_{1}$ and $\phi_{2}$ are the predictions on coarse and fine grids accordingly. 
Multiphase mixing was simulated in Eulerian-Eulerian model according to which the phases may interpenetrate each other. Five impeller revolutions per second produce fully turbulent flow (24000 Re). The k- $\varepsilon$ Realizable model was used for turbulence modeling since it was designed to overcome the difficulties of the Standard k- $\varepsilon$ model when rotation and/or swirling are involved via the introduction of turbulence viscosity as a variable instead of being constant [16]. The impeller motion was modelled via the MRF approach where the fluid in the region surrounding the rotating parts moves around a "frozen" impeller and a shaft. By producing boundary conditions for the interfacing walls between the zones, the motion transfers to the outer stationary zone.

To avoid computational instabilities, mixing of the liquid phase only was simulated first until convergence was reached and then the gas flow rate was activated with a constant bubble size of $1 \mathrm{~mm}$. The reactor top was assigned as pressure outlet with backflow volume fraction of air set to zero, which corresponds to degassing condition. The interphase interaction included only the drag force since the cumulative effect of lift, wall lubrication and turbulence diffusion forces can be considered negligible according to Lane et al. and Scargiali et al. [17], [18]. Scargiali et al. and Brucato et al. [19] showed the superior performance of the Morsi-Alexander drag force in combination with the Brucato modification coefficient included. Originally, the Brucato coefficient was suggested for solid particles being later reduced by two orders of magnitude to adopt it for gas bubbles [14], [19].

Third order differencing QUICK (Quadratic Upwind Interpolation for Convective Kinetics) scheme was used for momentum and turbulence because of its higher accuracy. Coupling of pressure and velocity was performed by using the Semi-Implicit algorithm. The simulations were continued until the residuals of all the variables were lower than $10^{-4}$. Two-phase simulations were run until both the convergence and the error in the air mass balance between the inlet and the outlet reached acceptable level of $5 \%$.

Single phase mixing computation took around 8500 iterations, which corresponds to 14 hours of real time when four cores were involved (Intel(R) Xeon(R) w3565 $3.2 \mathrm{GHz}$ and 24 Gb RAM) Multiphase simulations took no more than 18000 iteration and 30 hours of real time.

\section{Averaging Procedure}

The reactor construction and the four baffles against the six blades of Rushton turbine allow positioning the impeller within angles between $0^{\circ}$ to $60^{\circ}$ with respect to the baffles. The simulations were done at four positions of the impeller $0^{\circ}$, $15^{\circ}, 30^{\circ}$, and $45^{\circ}$ correspondingly, as it is depictured in Fig. 3. Then, the velocity profiles representing the different regions of the reactor were sampled from PIV and CFD results and averaged in Matlab over the different combinations of the impeller positions. The resulting data of the single phase and multiphase mixing are plotted versus the PIV tests in Fig. 4-Fig. 10. The major complication in the averaging is to make the data acquisition when the number and the shape of the elements differ as the impeller turns. In this work, all the data correlation was accomplished manually. However, it is recommended that in future automatic CFD tools for averaging should be created.

\section{E. Single Phase Averaging Assessment}

Before the averaging results are compared to the experimental data, it is interesting to see the velocity component profiles at different positions along the reactor height. The vessel may be split into three regions as follows: the bottom, impeller and reactor upper part regions. The profiles have been chosen so that all the three parts are tested. The results are gathered in Fig. 4 and Fig. 5.

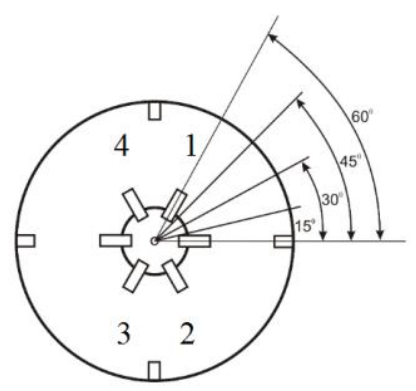

Fig. 3. Impeller positioning diagram.

\section{RESULTS}

The major difference in the velocity components can be seen at the 35,65 and $365 \mathrm{~mm}$ profiles whereas the 125,155 and $265 \mathrm{~mm}$ ones indicate no dispersion. The rotating zone expectedly produces the highest velocity variation as how in the $125 \mathrm{~mm}$ profile. The MRF technique employs averaging at the interface walls before the motion is transferred to the stationary zone. By careful examination of the variation of the velocity components along the sampled profiles, it can be noticed that the biggest deviation is between the angles $0^{\circ}$ and $30^{\circ}$, which signify that these two are the extreme impeller positions. In addition, some deviation may be noticed over the distance between the bottom and the top parts of the tank.

The RANS models are known to produce accurate results near the source of energy. In the profiles at $65,125,155 \mathrm{~mm}$ the simulation results are indeed qualitatively in the best agreement with the experimental ones. The RANS models have difficulties with flow separation and swirling predictions and they are greatly dependent on the domain geometry and grid configuration. The round bottom and the position of the baffles create difficulties for prediction of hydrodynamics in the bottom part, as represented by the $35 \mathrm{~mm}$ profile. In the regions that are far from the source of energy, the agreement is very poor.

It is important to know the sufficient number of samples that should be used when averaging the data. Therefore, it was decided to use the two extreme impeller positions, for example $0^{\circ}$ and $30^{\circ}$, and all four samples for testing purposes. The outputs are presented in Fig. 6. The biggest deviation can be observed in the close vicinity of the impeller in the rotating zone, which is shown in the $65 \mathrm{~mm}$ profile. Therefore, it is recommended to do the averaging of the results over all the four angles of the agitator positions when this particular zone is crucial in the research. What comes to the top and the bottom parts, averaging over all positions is not that necessary and in single phase mixing simulation of the two extreme impeller locations produces similar results. 

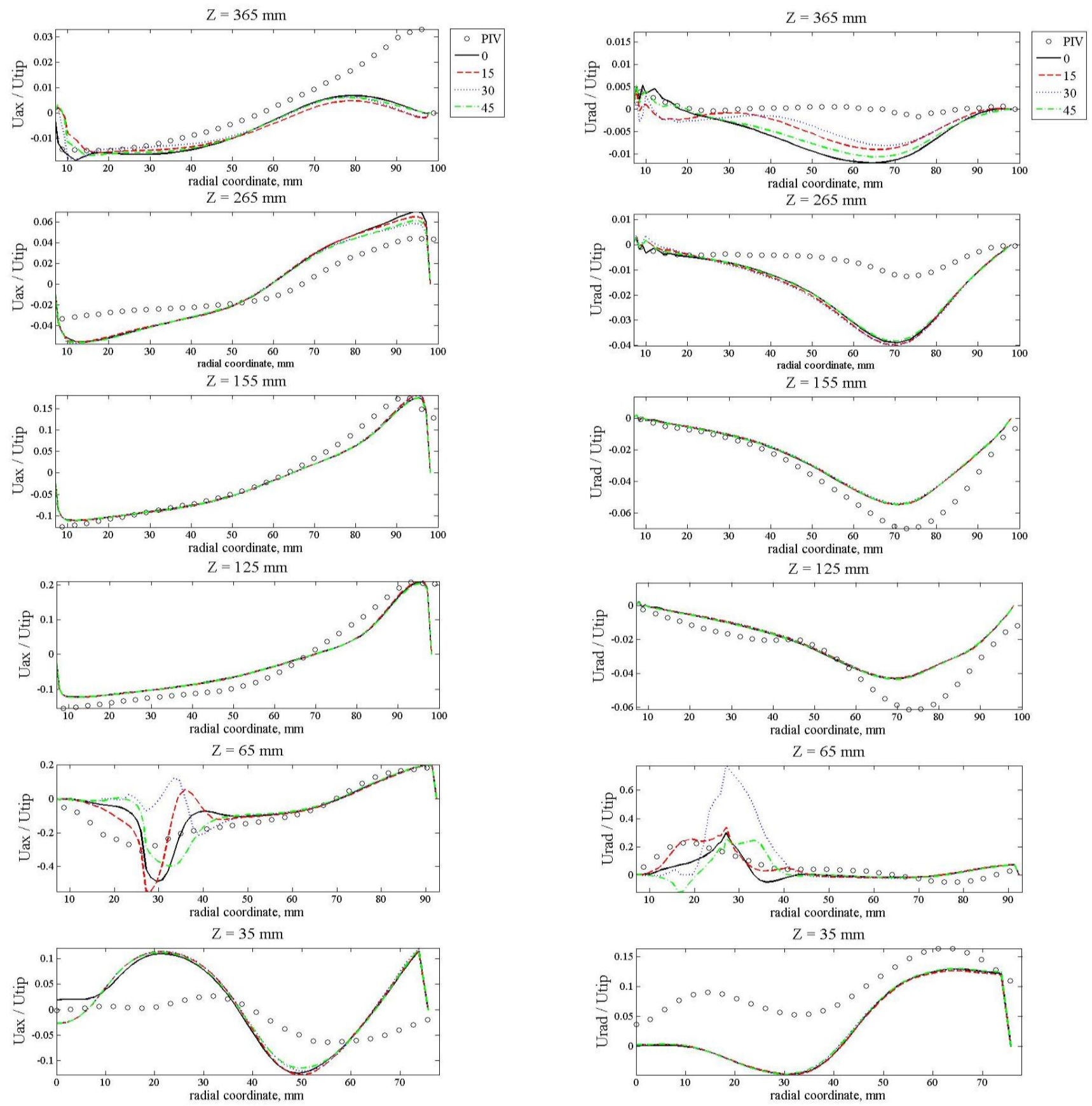

Fig. 4. Comparison of axial velocity profiles at different impeller positions produced by averaged results from CFD and PIV.

Fig. 5. Comparison of radial velocity profiles at different impeller positions produced by averaged results from CFD and PIV.

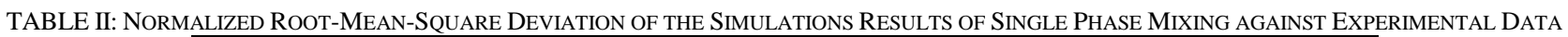

\begin{tabular}{|c|c|c|c|c|c|c|c|c|c|c|c|c|}
\hline \multirow{3}{*}{$\begin{array}{l}\text { Angle } \\
\text { of impeller } \\
\text { position }\end{array}$} & \multicolumn{12}{|c|}{ Normalized RMS deviation of radial and axial velocity components along the sampled profiles, $\%$} \\
\hline & \multicolumn{2}{|c|}{$35 \mathrm{~mm}$} & \multicolumn{2}{|c|}{$65 \mathrm{~mm}$} & \multicolumn{2}{|c|}{$125 \mathrm{~mm}$} & \multicolumn{2}{|c|}{$155 \mathrm{~mm}$} & \multicolumn{2}{|c|}{$265 \mathrm{~mm}$} & \multicolumn{2}{|c|}{$365 \mathrm{~mm}$} \\
\hline & radial & axial & radial & axial & radial & axial & radial & axial & radial & axial & radial & axial \\
\hline $0^{\circ}$ & 68.1 & 194.8 & 71.1 & 42.67 & 26.2 & 26.0 & 27.0 & 20.2 & 203.5 & 62.8 & 609.0 & 72.3 \\
\hline $15^{\circ}$ & 68.3 & 202.31 & 46.6 & 51.3 & 26.2 & 25.2 & 27.6 & 19.2 & 212.5 & 60.7 & 428.2 & 81.3 \\
\hline $30^{\circ}$ & 67.5 & 201.7 & 116.6 & 81.4 & 26.3 & 25.6 & 28.1 & 19.0 & 216.3 & 57.3 & 383.4 & 77.3 \\
\hline $45^{\circ}$ & 67.4 & 194.7 & 89.9 & 47.0 & 25.7 & 26.1 & 27.4 & 20.0 & 200.6 & 57.5 & 531.6 & 75.7 \\
\hline Averaging over & & & & & & & & & & & & \\
\hline $0^{\circ}$ and $30^{\circ}$ & 67.9 & 198.4 & 76.9 & 47.3 & 26.2 & 25.8 & 27.5 & 19.6 & 209.8 & 59.7 & 486.9 & 78.4 \\
\hline all four angles & 67.8 & 198.2 & 52.7 & 32.5 & 26.1 & 25.7 & 27.3 & 19.5 & 208.1 & 59.4 & 481.0 & 76.2 \\
\hline
\end{tabular}




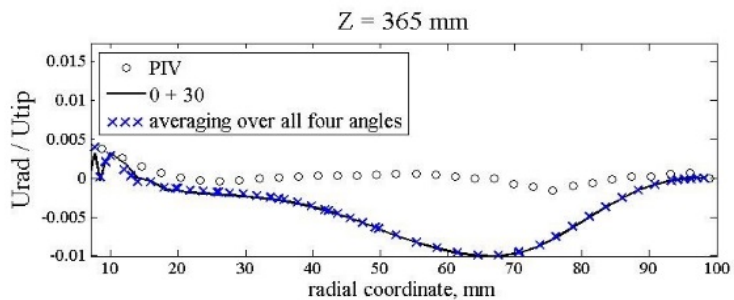

$\mathrm{Z}=65 \mathrm{~mm}$

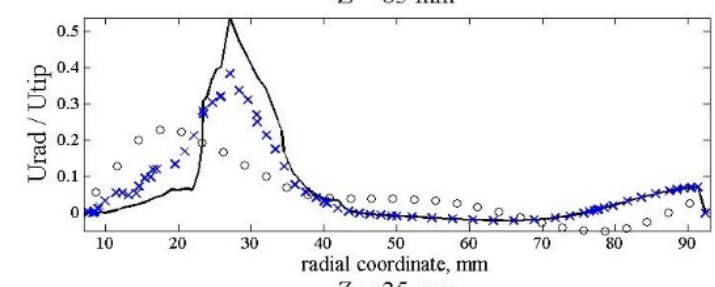

$\mathrm{Z}=35 \mathrm{~mm}$

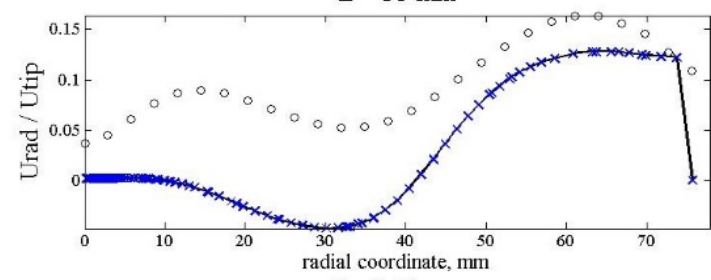

$\mathrm{Z}=365 \mathrm{~mm}$

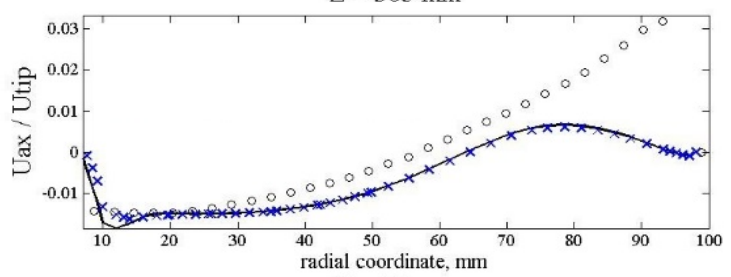

$\mathrm{Z}=65 \mathrm{~mm}$

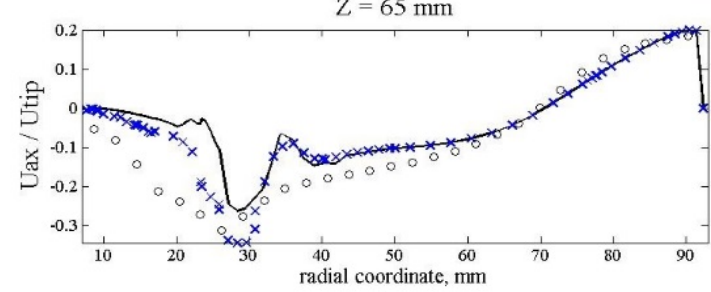

$\mathrm{Z}=35 \mathrm{~mm}$

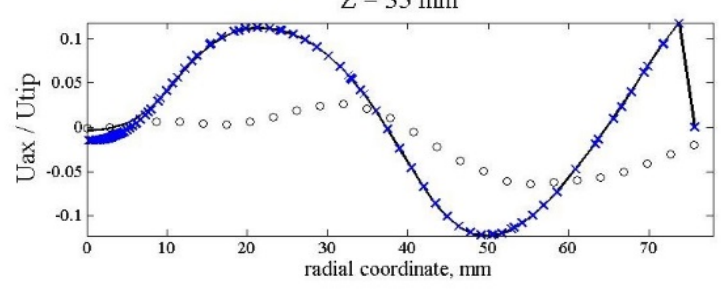

Fig. 6. Axial and radial velocity profiles comparison of the CFD simulations averaged over different impeller positions and the PIV results.

Quantitative evaluation of improvement achieved by averaging of simulation results is of great interest and may also give valuable information about how the number of samples taken into account may improve the simulation results in comparison to experimental data. The quantitative analysis for single phase is presented in Table II. The normalized root-mean-square error shows the deviation of the simulation results from the experimental data. It shows the influence of the impeller position on the overall hydrodynamics in the reactor especially in the regions around the stirrer and in the top of the vessel near the gas-liquid surface. The influence would not be that much if the aspect ratio of the vessel would not be high which is clear by considering the profiles at 155 and $265 \mathrm{~mm}$.
Benefits of the averaging procedure can be estimated from Table II. Obviously, a decreasing tendency in deviation can be tracked as more impeller angles are involved. The biggest improvement can be seen at the profiles 65 and $365 \mathrm{~mm}$ where the simulation results deviate the most. As it is shown in Fig. 3, the six-blade-impeller vs. four-baffle system repeats itself after every $60^{\circ}$ of rotation. Thus, $15^{\circ}$ plane of rotation in sector 1 where the deviation is at minimum (46.6\%) corresponds to the $30^{\circ}$ plane of blade location in sector 4 where the deviation is at maximum $(116.6 \%)$.

Nevertheless, averaging of the results at the two extreme positions increases accuracy significantly. As more angles are involved, the deviation from experimental data reduces further but not drastically. Therefore, it is reasonable to perform at least two simulations of stirrer tank with the impeller position at the two utmost locations in order to have more accurate hydrodynamics of single phase mixing.

\section{Multiphase Averaging Assessment}

The averaging procedure is of particular interest and importance for multiphase systems. Gas-liquid mixing was considered next and the influence of the impeller position relative to the baffles was studied by comparing the velocity profiles at different locations. It must be noted that multiphase mixing is a complicated phenomenon that includes multiple counter acting forces and the simulation of its hydrodynamics has greater uncertainty when compared to experimental results than that of the single phase.

The profiles that are shown below in Figs. 7 - 10 represent the complete reactor from the top to bottom and from the vessel center, or agitator shaft, to the tank wall. The whole reactor lay-out is easily visualized in the presented manner when looking at the profiles and assessing qualitatively the hydrodynamics in the reactor. Since the multiphase system is complicated with interfacial forces and turbulence computed per phase, even a slight change in the impeller blade position gives deviation in the velocity components in every sampled profile. As in the case of the single phase, the biggest variation is observed near the impeller.

One may note that the angle of the agitator with respect to baffles has a bigger effect on the gaseous phase. In the current work, the gas flow rate is rather low and therefore, the primary phase turbulence has great impact on the movement of the secondary phase. Thus, it is important to undertake averaging in order to produce results of higher accuracy.

In addition, it could be expected that at higher gas flow rates gas hydrodynamics should be less dependent on the fluctuations in the liquid flow and therefore, averaging over the two extreme positions for gas phase should be enough. However, the number of impeller positions to be involved in the averaging is dictated by the qualitative evaluation of hydrodynamics (see Table III and Table IV).

Analysis of the root-mean square deviation of multiphase nixing is less straightforward due to the complexity of gas-liquid interaction modeling in fully turbulent flow. And as an outcome, the accuracy raise after averaging procedure is less than in single phase mixing. 

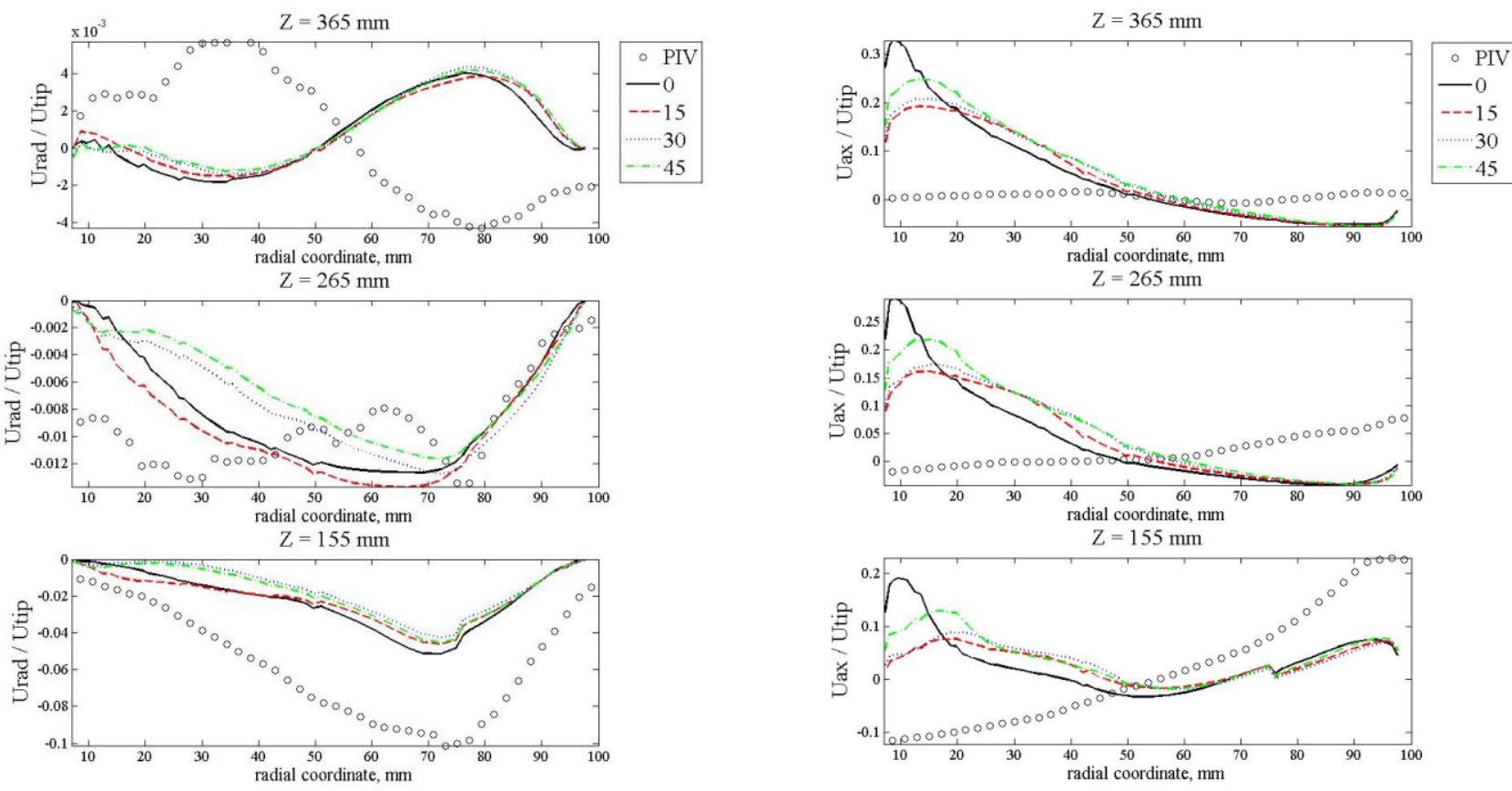

$\mathrm{Z}=125 \mathrm{~mm}$
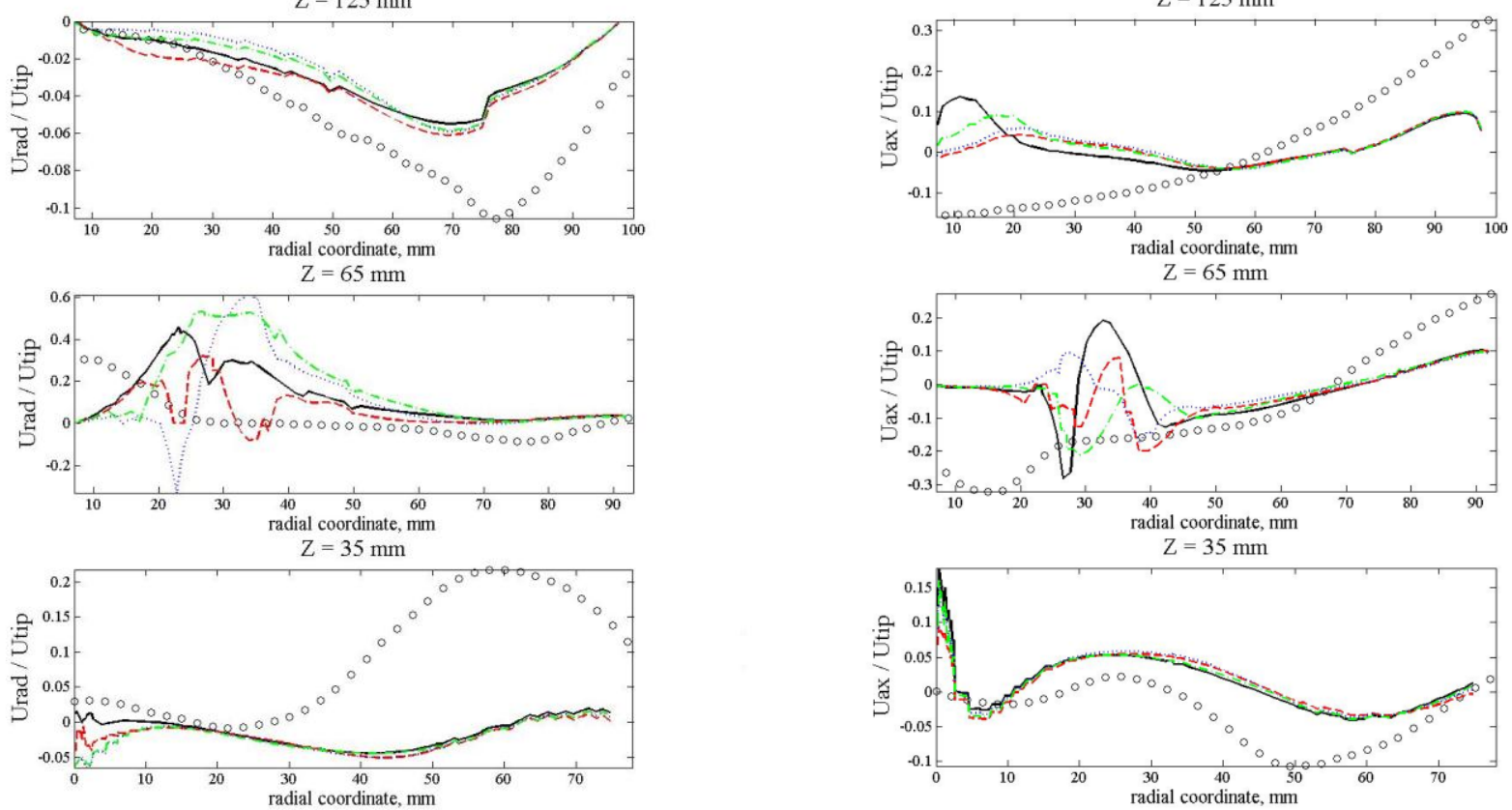

Fig. 7. Radial velocity profiles comparison of the CFD simulations in liquid phase averaged over different impeller positions and the PIV results

Fig. 8. Axial velocity profiles comparison of the CFD simulations in liquid phase averaged over different impeller positions and the PIV results.

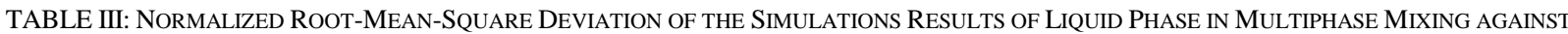
EXPERIMENTAL DATA

\begin{tabular}{|c|c|c|c|c|c|c|c|c|c|c|c|c|}
\hline \multirow{3}{*}{$\begin{array}{l}\text { Angle } \\
\text { of impeller } \\
\text { position }\end{array}$} & \multicolumn{12}{|c|}{ Normalized RMS deviation of radial and axial velocity components along the sampled profiles, $\%$} \\
\hline & \multicolumn{2}{|c|}{$35 \mathrm{~mm}$} & \multicolumn{2}{|c|}{$65 \mathrm{~mm}$} & \multicolumn{2}{|c|}{$125 \mathrm{~mm}$} & \multicolumn{2}{|c|}{$155 \mathrm{~mm}$} & \multicolumn{2}{|c|}{$265 \mathrm{~mm}$} & \multicolumn{2}{|c|}{$365 \mathrm{~mm}$} \\
\hline & radial & axial & radial & axial & radial & axial & radial & axial & radial & axial & radial & axial \\
\hline $0^{\circ}$ & 215.7 & 122.1 & 71.7 & 96.7 & 123.2 & 111.2 & 65.5 & 129.6 & 39.8 & 407.3 & 130.2 & 1331.5 \\
\hline $15^{\circ}$ & 218.0 & 111.0 & 84.4 & 71.8 & 136.2 & 99.1 & 68.1 & 117.5 & 35.0 & 357.4 & 130.1 & 1112.5 \\
\hline $30^{\circ}$ & 223.0 & 120.4 & 78.8 & 73.8 & 114.1 & 104.6 & 72.5 & 124.2 & 44.4 & 377.5 & 131.3 & 1171.7 \\
\hline $45^{\circ}$ & 211.8 & 117.4 & 70.7 & 80.7 & 118.7 & 111.0 & 70.4 & 133.4 & 50.5 & 420.0 & 225.8 & 206.0 \\
\hline Averaging over & & & & & & & & & & & & \\
\hline $0^{\circ}$ and $30^{\circ}$ & 218.5 & 120.1 & 68.0 & 73.5 & 118.2 & 106.6 & 68.8 & 124.9 & 41.6 & 385.2 & 130.6 & 1235.9 \\
\hline all four angles & 216.6 & 117.4 & 57.3 & 71.3 & 115.6 & 105.5 & 68.3 & 124.5 & 41.4 & 385.2 & 129.8 & 1102.3 \\
\hline
\end{tabular}



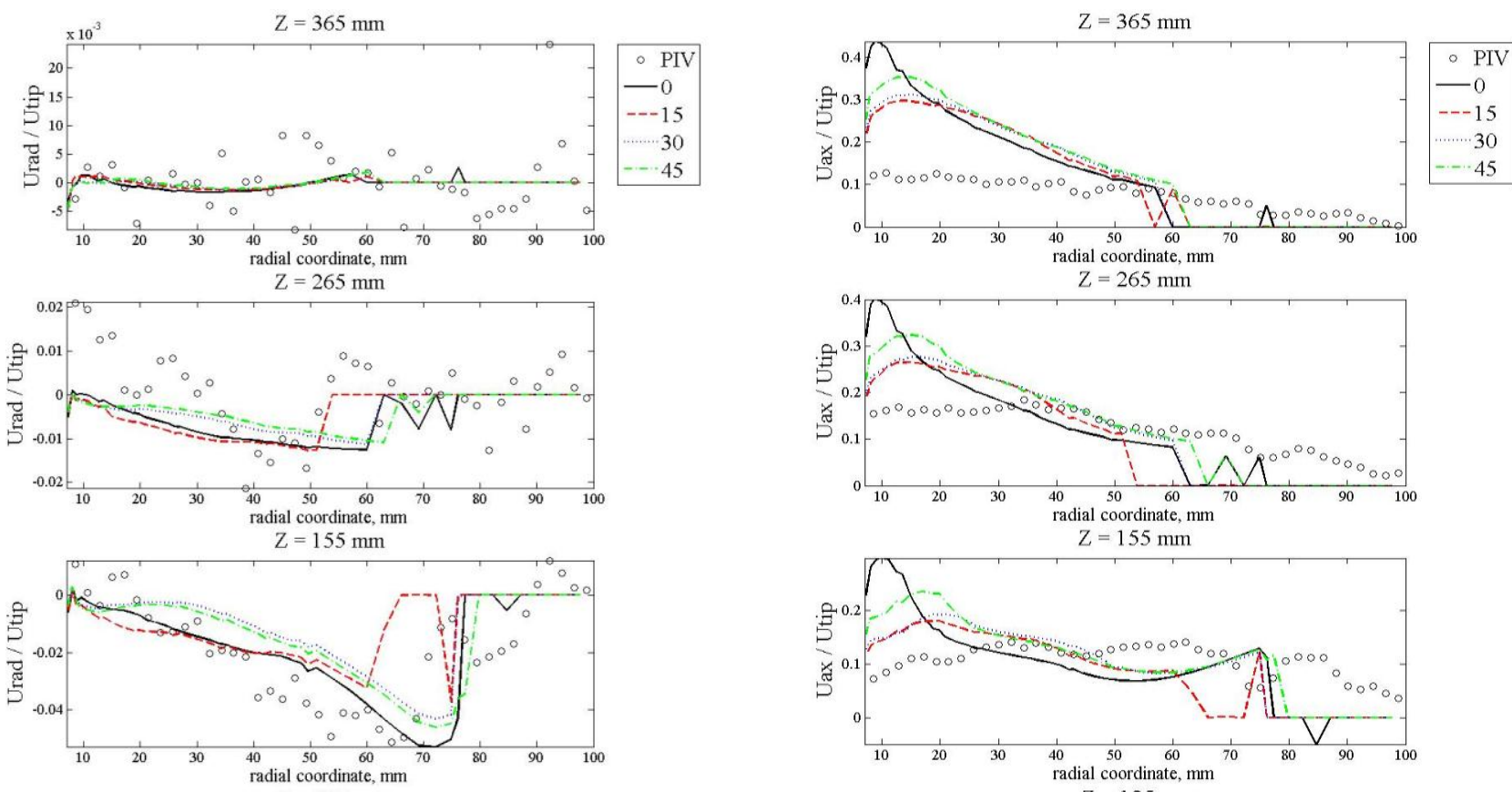

$\mathrm{Z}=125 \mathrm{~mm}$
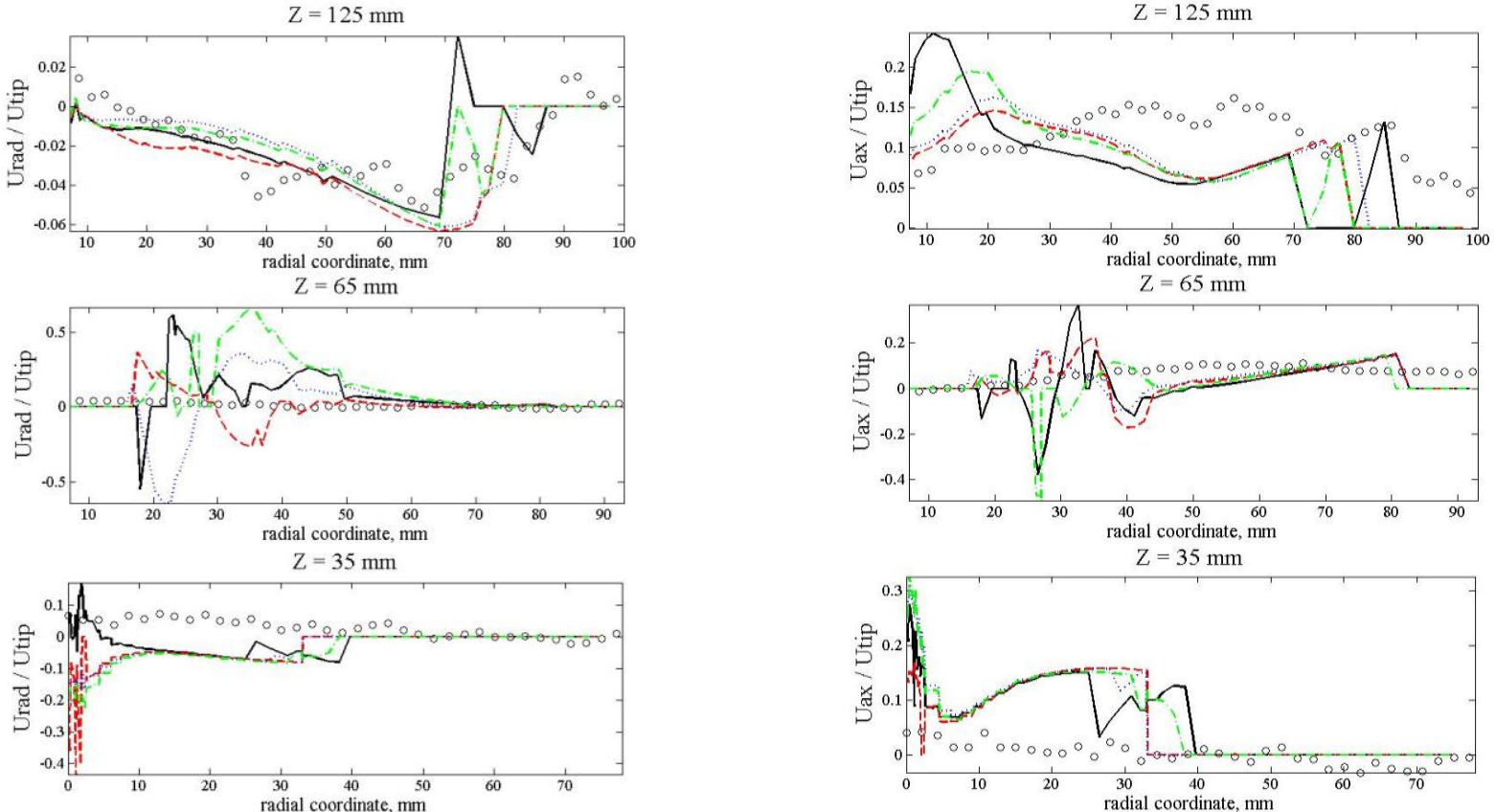

Fig. 9. Radial profiles comparison of the CFD simulations in gas phase averaged over different impeller positions and the PIV results.

Fig. 10. Axial velocity profiles comparison of the CFD simulations in gas phase averaged over different impeller positions and the PIV results.

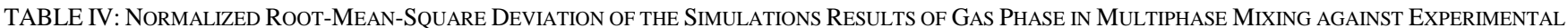
DATA

\begin{tabular}{|c|c|c|c|c|c|c|c|c|c|c|c|c|}
\hline \multirow{3}{*}{$\begin{array}{c}\text { Angle } \\
\text { of impeller } \\
\text { position }\end{array}$} & \multicolumn{12}{|c|}{ Normalized RMS deviation of radial and axial velocity components along the sampled profiles, \% } \\
\hline & \multicolumn{2}{|c|}{$35 \mathrm{~mm}$} & \multicolumn{2}{|c|}{$65 \mathrm{~mm}$} & \multicolumn{2}{|c|}{$125 \mathrm{~mm}$} & \multicolumn{2}{|c|}{$155 \mathrm{~mm}$} & \multicolumn{2}{|c|}{$265 \mathrm{~mm}$} & \multicolumn{2}{|c|}{$365 \mathrm{~mm}$} \\
\hline & radial & axial & radial & axial & radial & axial & radial & axial & radial & axial & radial & axial \\
\hline $0^{\circ}$ & 107.8 & 112.7 & 580.4 & 472.4 & 44.6 & 105.6 & 34.8 & 97.0 & 134.5 & 91.9 & 84.8 & 511.5 \\
\hline $15^{\circ}$ & 110.2 & 122.4 & 334.3 & 394.5 & 58.9 & 98.2 & 38.2 & 81.8 & 144.6 & 79.4 & 85.1 & 432.6 \\
\hline $30^{\circ}$ & 109.9 & 122.9 & 759.6 & 329.5 & 47.7 & 97.1 & 39.2 & 80.5 & 122.7 & 75.6 & 83.7 & 450.9 \\
\hline $45^{\circ}$ & 109.4 & 116.8 & 879.9 & 356.4 & 48.7 & 98.6 & 36.8 & 81.9 & 115.9 & 76.0 & 154.5 & 116.0 \\
\hline Averaging over & & & & & & & & & & & & \\
\hline $0^{\circ}$ and $30^{\circ}$ & 109.8 & 119.5 & 546.4 & 330.0 & 48.9 & 100.1 & 34.9 & 86.8 & 128.2 & 82.5 & 84.2 & 475.9 \\
\hline all four angles & 109.3 & 118.6 & 427.3 & 328.9 & 45.2 & 98.9 & 34.1 & 83.8 & 128.0 & 79.6 & 84.0 & 455.9 \\
\hline
\end{tabular}


In multiphase mixing qualitative analysis of liquid phase shows higher values for root-mean-square deviations at all profiles taken. Analogously to single phase mixing, the biggest deviation of simulation appears near the impeller and, therefore, averaging is important in that region. Deviations in gas phase are slightly less dependent on impeller position in modeling. The profile at $65 \mathrm{~mm}$ is an exception due to the radial flow generated by the mixer is dominant in this case.

The amount of impeller position needed to get improved simulation accuracy is similar to single phase mixing modeling. The larger the number of the stirrer positions taken into account, the higher the accuracy in the results one may achieve. However, improvement in accuracy gradually decreases when more impeller positions are added. Therefore, one should find a compromise between the accuracy needed and the affordable computational time.

\section{CONCLUSION}

The results in the presented work clearly indicate that the averaging of results improves the accuracy of CFD simulations of single and multiphase mixing in a baffled stirred tank when the "frozen rotor" approach is applied. It was observed that the profiles of the velocity components are various depending on the impeller angle relative to the baffles. Such variation is low for single phase and significantly higher when a secondary gaseous phase is introduced. It was proved that averaging over the two utmost impeller positions improves the simulated results of hydrodynamics. As far as the Rushton turbine is concerned, averaging over four impeller positions produces more accurate results than just over the two extreme stirrer positions. Researchers and engineers, who deal with stirred tank modeling, are advised to execute several simulations with the stirrer turned within the sector where stirrer-blade-baffle interaction appears. Such a sector is dictated by the number of impeller blades relative to the quantity and construction of baffles. The proposed above suggestion is just for modeling of single phase mixing as well as for multiphase mixing. Since the "frozen rotor" technique is widely used in mixing simulations, it makes sense to consider developing automatic or semiautomatic methods and tools for averaging procedure.

\section{REFERENCES}

[1] V. R. Vivek, "CFD predictions of flow near impeller blades in baffled stirred vessels: assessment of computational snapshot approach," Chemical Engineering Communications, vol. 189, pp. 895-922, 2002.

[2] B. Bai, Y. Wang, and P. M. Armenante, "Velocity profiles and shear strain rate variability in the USP dissolution testing apparatus 2 at different impeller agitation speeds," International Journal of Pharmaceutics, vol. 403, pp. 1-14, 2010.

[3] W. Huang and K. Li, "CFD simulation of flows in stirred tank reactors," Nuclear Reactor Thermal Hydraulics and Other Applications, InTech, 2013, ch. 5.

[4] R. I. Issa and A. D. Gosman, "The computation of three-dimensional turbulent two-phase flows in mixer vessels," in Proc. 2nd International Conference of Numerical Methods in Laminar and Turbulent Flow, 1981, p. 829.

[5] P. S. Harvey and M. Greaves, "Turbulent-flow in an agitated vessel.1. A predictive model," Transactions of the Institution of Chemical Engineers, vol. 60, no. 4, pp. 195-200, 1982

[6] G. L. Lane, M. P. Schwarz and G. M. Evans "Modeling of the Interaction between gas and liquid in stirred vessels," in Proc. 10th European Conference on Mixing, 2000, pp. 197-204.
[7] M. Laakkonen, P. Moilanen, V. Alopaeus, and J. Aittamaa, "Local bubble size distributions, gas-liquid interfacial area and gas holdups in stirred vessel with particle image velocimetry," Chemical Engineering Journal, vol. 109, pp. 37-47, 2005

[8] J. Aubin, S. M. Kresta, J. Bertrand, C. Xuere, and D. F. Fletcher, "Alternate operating methods for improving the performance of a continuous stirred tank reactor," Trans IChemE, vol. 84, pp. 569-582, 2006

[9] V. N. Vlachakis, "Turbulent characteristics in stirring vessels: A numerical investigation," M.S. Thesis in Mech. Eng., Blacksburg, Virginia, June 16, 2006

[10] M. Marshall and A. Bakker, "Computational Fluid Mixing," Fluent Inc., Lebanon, 2002

[11] K. D. Jensen, "Flow measurements," Journal of the Brazilian Society of Mechanical Science \& Engineering, Vol. XXVI, No. 4, pp. 400-419, 2004

[12] H. Wu and G. K. Patterson, "Laser-doppler measurements of turbulent flow parameters in a stirred mixer," Chemical Engineering Science, vol. 44, no. 10, pp. 2207-2221, 1989

[13] L. Xinhong, B. Yuyun, L. I. Zhipeng, and G. Zhengming, "Analysis of turbulence structure in the stirred tank with a deep hollow blade disc turbine by time-resolved PIV," Fluid Flow and Transport Phenomena, vol. 18 , no. 4, pp. 588-599, 2010

[14] M. Laakkonen, "Development and validation of mass transfer models for the design of agitated gas-liquid reactors" PhD Thesis in Tech., Espoo, Finland, 2006

[15] H. Wang and Z. Zhai, "Analyzing grid independency and numerical viscosity of computational fluid dynamics for indoor environment applications," Building and Environment Journal, vol. 52, pp. 107-118, 2012.

[16] T. H. Shih, W. W. Liou, A. Shabbir, Z. Yang, and J. Zhu, "A new k-8 eddy viscosity model for high reynolds number turbulent flows - model development and validation," Computers Fluids, vol. 24, no. 3, pp. 227-238, 1995

[17] G. L. Lane, M. P. Schwarz, and G. M. Evans, "Modeling of the Interaction between gas and liquid in stirred vessels," in Proc. 10th European Conference on Mixing, pp. 197-204, 2000

[18] F. Scargiali, A. D'Orazio, F. Grisafi, and A. Brucato, "Modeling and simulation of gas-liquid hydrodynamics in mechanically stirred tanks," Chemical Engineering Research and Design, vol. 85, no. A5, pp. 647-653, 2007

[19] A. Brucato, F. Grisafi, and G. Montante, "Particle drag coefficients in turbulent fluids," Chemical Engineering Science, vol. 53, no. 18, pp. 3295-3314, 1998.

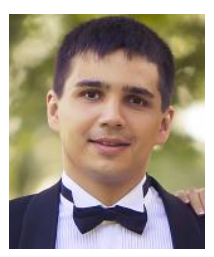

Dmitry Gradov is a doctoral student at Lappeenranta University of Technology (LUT). He received his first MSc (2010) in mineral processing from Saint-Petersburg State Mining University and second MSc (2011) from LUT in the field of product and process development. He has been a doctoral student since 2012. His current research is related to numerical modeling and experimental study of multiphase hydrodynamics in batch stirred tank.

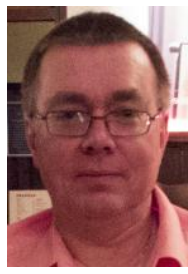

Arto Laari is a research scientist at Lappeenranta University of Technology (LUT). He received his $\mathrm{PhD}$ (2005) and the title of Docent (2010) from LUT in the field of product and process development. He has been working as an associate professor in the same department in 2010-2014. His current scientific interests are in the development of sustainable metal leaching processes and in the storage and transformation of renewable energy.

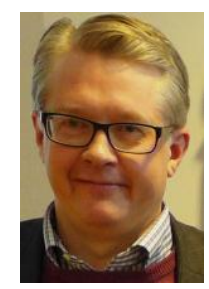

Tuomas Koiranen is a professor at Lappeenranta University of Technology (LUT). He received his PhD (1998) in Helsinki University of Technology, and the title of Docent (2004) from Lappeenranta University of Technology. After having 12 years industrial experience in pharmaceutical R\&D as research scientist, he started the professorship at Lappeenranta University of Technology in 2013. His current scientific and teaching interests are fluid dynamics in chemical engineering applications, liquid droplet behavior in reactive flows, process development and intensified processes. 